\title{
Accounting for Multiple Scattering in Retrievals from Space Lidar
}

\author{
Dave Winker \\ Atmospheric Sciences, NASA Langley Research Center \\ Hampton, Virginia
}

\begin{abstract}
Multiple scattering effects on signals from space lidar are significant and must be accounted for in the retrieval of aerosol and cloud extinction. It is shown that a simple parameterization of multiple scattering allows one to account for multiple scattering effects using a slightly modified form of a commonly used single-scatter retrieval solution. All orders of scattering are considered in the development of parameterizations appropriate for the CALIPSO lidar. Applications to aerosol and cloud retrievals are discussed.
\end{abstract}

Keywords: Spaceborne lidar, multiple scattering, aerosols, clouds, extinction, CALIPSO

\section{INTRODUCTION}

Lidar retrievals are most often based on a solution of the classic lidar equation, which is a single-scatter approximation that ignores higher order (multiple) scattering ${ }^{1,2,3}$. Multiple scattering can alter the apparent extinction or transmittance of the medium, can produce depolarization of the return signal, and can produce stretching of the return pulse. For most lidar systems the magnitude of the multiply-scattered signal is so small these effects are insignificant and can often be ignored without introducing significant errors. Multiple scattering effects can be very significant for satellite lidar systems, however, due to the large footprints typical of space geometries. For the same field of view, the footprint diameter of a satellite lidar is roughly two orders of magnitude larger than for ground-based or airborne lidars, due to the large distance from the atmosphere, allowing a much greater fraction of the multiply-scattered light to contribute to the return signal. Because of this, an algorithm intended to retrieve extinction from satellite lidar data must explicitly account for multiple scattering effects on the return signal. This issue was first encountered by the Lidar In-space Technology Experiment (LITE), which flew on the Space Shuttle in 1994,5. The focus in this paper is on accounting for multiple scattering effects in the extinction retrieval algorithms being developed for CALIOP, the two-wavelength depolarization lidar which will fly on the CALIPSO satellite ${ }^{6}$.

The nature of the multiple scattering is fundamentally dependent on the scattering phase function of the atmospheric particles and on the sensing geometry of the lidar. The magnitude of the volume extinction coefficient and homogeneity of the scattering medium also play a role. When the scattering medium becomes highly turbid and the scattering approaches the diffusion regime, multiple scattering overwhelms the single-scattered component of the return signal and pulse stretching becomes significant. To fully understand the multiple scattering issue for space lidars, it is necessary to examine the multiply-scattered lidar return signal for a variety of particle types and a variety of scenarios.

Accurate calculation of the multiply-scattered return signal can be difficult. Analytic forms of the exact solution of the radiative transfer equation for the lidar geometry are generally intractable and the applicability of approximate solutions can be suspect. Instead, two different Monte Carlo codes have been developed to investigate multiple scattering issues. One is a fairly simple code to compute the intensity of the lidar return ${ }^{7}$. The other computes the full Stokes scattering matrix, which is required to model the polarization of the return signal ${ }^{8}$. Monte Carlo approaches allow the full physics to be incorporated but can be computationally intensie. Statistical techniques can be used to increase efficiency but involve approximations which can introduce errors. Another drawback is that the Monte Carlo approach does not 
provide an analytic formulation which can form the basis of a retrieval algorithm. In order to complement Monte Carlo studies of multiple scattering effects, a simple analytic algorithm has been developed to compute single- and doublescattering. A double-scattering calculation is fairly accurate for aerosols, but even in clouds it provides insight and can be used as a check on Monte Carlo codes.

As mentioned above, the multiple scattering effects can vary dramatically in nature and magnitude depending on the lidar sensing geometry and the characteristics of the target being sensed. Here we will focus on cirrus and aerosols, where the CALIOP measurements provide no direct information on multiple scattering and the effects must be modeled. Simulations show that pulse stretching in cirrus and aerosols is not significant. For dense water clouds in the boundary layer pulse stretching can be an issue and the lidar depolarization profile provides information on multiple scattering. Therefore a different retrieval approach is used for dense water clouds than that discussed below. The algorithm for retrieval of extinction profiles in aerosols and cirrus clouds will be presented, followed by a discussion of how the algorithm is applied in different circumstances.

\section{EXTINCTION RETRIEVAL AND THE PARAMETERIZATION OF MULTIPLE SCATTERING}

The extinction retrieval algorithm developed for CALIPSO ${ }^{9}$ is based on a two-component linear iterative technique ${ }^{1}$. The retrieval algorithm operates on a profile of attenuated backscatter, $\beta^{\prime}(r)$, produced by range-correcting and calibrating the raw return signal:

$$
\beta^{\prime}(r)=\left[\beta_{m}(r)+\beta_{p}(r)\right] T_{m}^{2}\left(r_{c}, r\right) \exp \left[-2 \eta(r) S_{p} \gamma_{p}\left(r_{c}, r\right)\right]
$$

where $m$ indicates the molecular component and $p$ the particulate (either cloud or aerosol) component, and $\gamma_{\mathrm{p}}$ represents the integration of the particulate backscatter coefficient between the calibration range $r_{c}$ and some range $r . T_{m}{ }^{2}\left(r_{c}, r\right)$ is the molecular transmission between $r_{c}$ and $r$. The particulate lidar ratio, $S_{\mathrm{p}}=\sigma_{\mathrm{p}} / \beta_{\mathrm{p}}$, may apply to either aerosol or cloud particles. Multiple scattering effects are parameterized as in Platt ${ }^{10}$ using a range-dependent multiple scattering function $\eta(r)$, defined as:

$$
\eta(\mathrm{z})=1-\operatorname{Ln}\left[\mathrm{P}_{\mathrm{TS}}(\mathrm{z}) / \mathrm{P}_{\mathrm{SS}}(\mathrm{z})\right] / 2 \tau(\mathrm{z})
$$

where TS and SS refer to the total scattered signal and the singly-scattered signal, respectively. The definition of $\eta(\mathrm{z})$ follows directly from the ratio of the single-scatter lidar return signal:

$$
\mathrm{P}_{\mathrm{SS}}(\mathrm{z})=\mathrm{C} \beta(\mathrm{z}) \exp (-2 \tau(\mathrm{z})) / \mathrm{R}^{2}
$$

and the multiply-scattered lidar return signal, expressed as:

$$
\mathrm{P}_{\mathrm{TS}}(\mathrm{z})=\mathrm{C} \beta(\mathrm{z}) \exp (-2 \eta(\mathrm{z}) \tau(\mathrm{z})) / \mathrm{R}^{2}
$$

The multiple scattering function $\eta(z)$ as defined in Equation (2) is in a convenient form to compute from the results of Monte Carlo simulations and is related in a simple way to an alternate multiple scattering function, $\mathrm{F}(\mathrm{z})$, originally used by Carnuth and Reiter ${ }^{11}$ :

$$
\mathrm{F}(\mathrm{z})=1-\eta(\mathrm{z})
$$

Given the calibrated return signal (1), the particulate backscatter is estimated by the retrieval algorithm as:

$$
\beta_{p}(r)=\beta^{\prime}(r) / T_{m}^{2}\left(r_{c}, r\right) \exp \left[-2 \eta(r) S_{p} \gamma_{p}\left(r_{c}, r\right)\right]-\beta_{m}(r)
$$


where $\gamma_{\mathrm{p}}\left(\mathrm{r}_{\mathrm{c}}, \mathrm{r}\right)$ is the integral of $\beta_{\mathrm{p}}(\mathrm{r})$ over range $r_{\mathrm{c}}$ to $r$. This equation is in a form appropriate for a forward solution, $r_{c}<$ $r$. The product of $\eta(\mathrm{r})$ and $S_{p}$ is written as $S_{p}{ }^{*}(r)$, the effective lidar ratio, which is range dependent. Given the value of $S_{p}{ }^{*}$, the backscatter profile can be retrieved iteratively. The algorithm can accommodate a value of $S_{p}{ }^{*}$ which varies with range. Once $\beta_{\mathrm{p}}(\mathrm{r})$ is retrieved, the particulate extinction profile is retrieved by scaling the retrieved backscatter profile by $S_{p}$ :

$$
\sigma_{\mathrm{p}}(\mathrm{r})=\mathrm{S}_{\mathrm{p}} \beta_{\mathrm{p}}(\mathrm{r})
$$

In many cases, $S_{p}{ }^{*}$ can be derived from the lidar measurement itself. $S_{p}$ can then be derived from $S_{p}{ }^{*}$ using a modeled value of $\eta(r)$.

In the next section, cloud and aerosol models appropriate for modeling multiple scattering are presented. Following that, the application of the retrieval algorithm under several different scenarios is presented.

\section{AEROSOL AND CLOUD MODELS}

For cirrus and aerosols, the CALIOP return signals contain no information on the degree to which they are affected by multiple scattering and the multiple scattering function must be estimated by modeling. Because $\eta(r)$ is dependent on the size, shape, and composition of the particles, the selection of realistic cloud and aerosol particle models is essential. All multiple scattering calculations assume the CALIOP viewing geometry: a receiver field of view of $130 \mu \mathrm{rad}$, a laser beam divergence of $100 \mu \mathrm{rad}$, and an orbit altitude of $705 \mathrm{~km}$.

\subsection{Aerosols.}

One of the functions of the CALIPSO data processing algorithms is to classify observed aerosol layers by type. The algorithm currently considers six aerosol types, each which is defined by an aerosol model from which the scattering phase function, $S_{a}$, and the multiple scattering function, $\eta(\mathrm{r})$, can be computed. Figure 1 shows phase functions corresponding to three of these aerosol types: two coarse-mode aerosols (sea salt and mineral dust) and one fine-mode aerosol (clean continental). The sea salt and clean continental phase functions are based on in situ measurements ${ }^{12}$. The

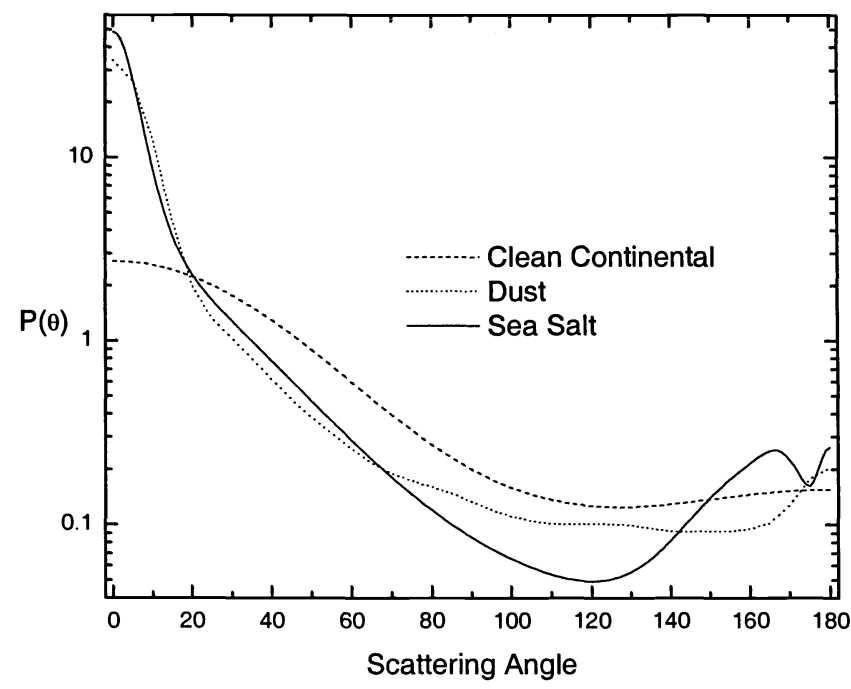

Figure 1. Phase functions corresponding to three aerosol models. 
phase function for dust was computed theoretically, based on realistic sizes, compositions, and shapes ${ }^{13}$. Figure 2 shows the growth of the multiply-scattered signal relative to the single-scattered signal as a function of range into an aerosol layer and that the relative strength of the multiply-scattered signal increases with increasing aerosol extinction. Figure 3 indicates the rationale for parameterization of multiple scattering in terms of $\eta(\mathrm{z})$. In this figure, for two aerosol types,

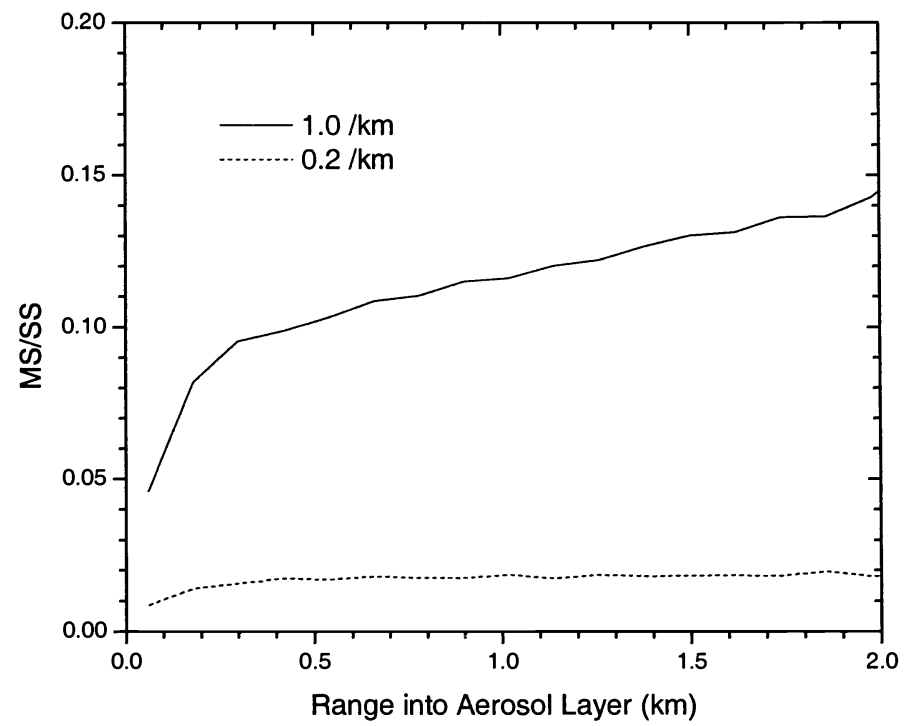

Figure 2. Ratio of aerosol multiple scattering to single scattering for two extinction coefficients, clean continental model.

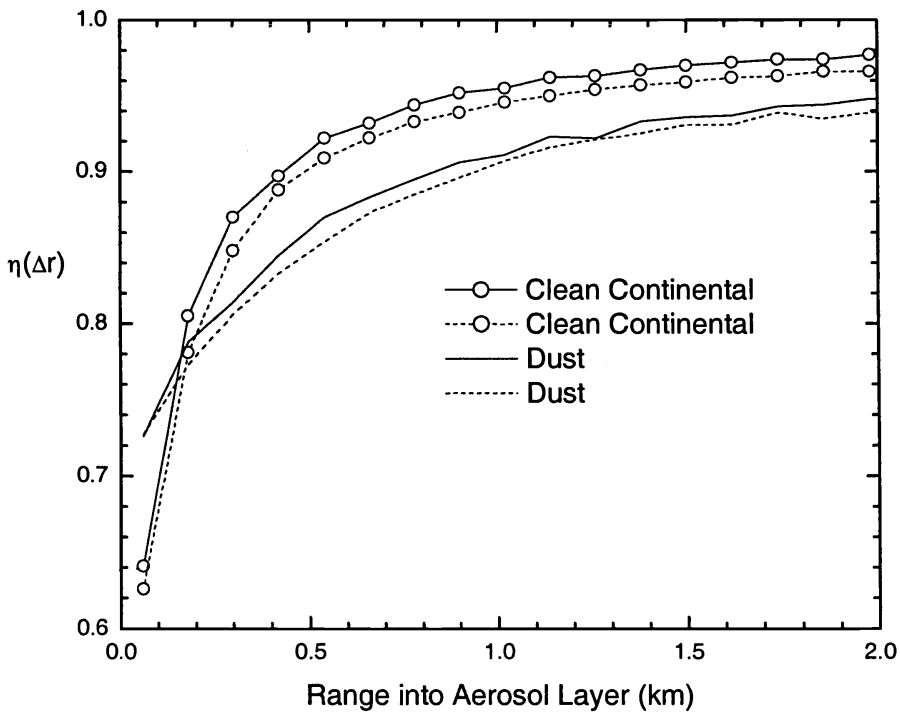

Figure 3. Aerosol multiple scattering functions vs. extinction: $0.2 / \mathrm{km}$ (solid); $1.0 / \mathrm{km}$ (dashed) 
we see that $\eta(z)$ is only a weak function of extinction cross-section. Thus, parameterizing multiple scattering using $\eta(z)$, which modifies the aerosol optical depth, has practical advantages over the approach of modeling multiple scattering as an additive term which increases the backscatter coefficient: $\beta(z)(1+Q(z))$. The dependence of $\eta(z)$ on the magnitude of the extinction is small enough that a single function can be used for each aerosol type, independent of extinction.

Figure 4 shows the multiple scattering functions corresponding to the three aerosol phase functions in Figure 1, for an extinction coefficient of $0.2 / \mathrm{km}$. The multiple scattering function is a strong function of range. However, for deep layers $\eta(z)$ approaches unity so that the multiple scattering has little effect on the optical depth retrieved. In this case the primary effect of multiple scattering is to reduce the apparent extinction at the top of the layer and alter the shape of the extinction profile. These results suggest that the multiple scattering function may depend primarily on aerosol size rather than other properties. Two-wavelength CALIOP returns provide information on whether the aerosol is dominated by coarse or fine mode, allowing a selection of the appropriate $\eta(r)$ function. In any case, considering that aerosol layers are usually greater than 500 meters deep, $\eta(r)$ is greater than 0.85 and so is not a large correction. The uncertainty in aerosol optical depth due to the estimation of $\eta(\mathrm{r})$ is probably less than that due to uncertainties in $S_{a}$.

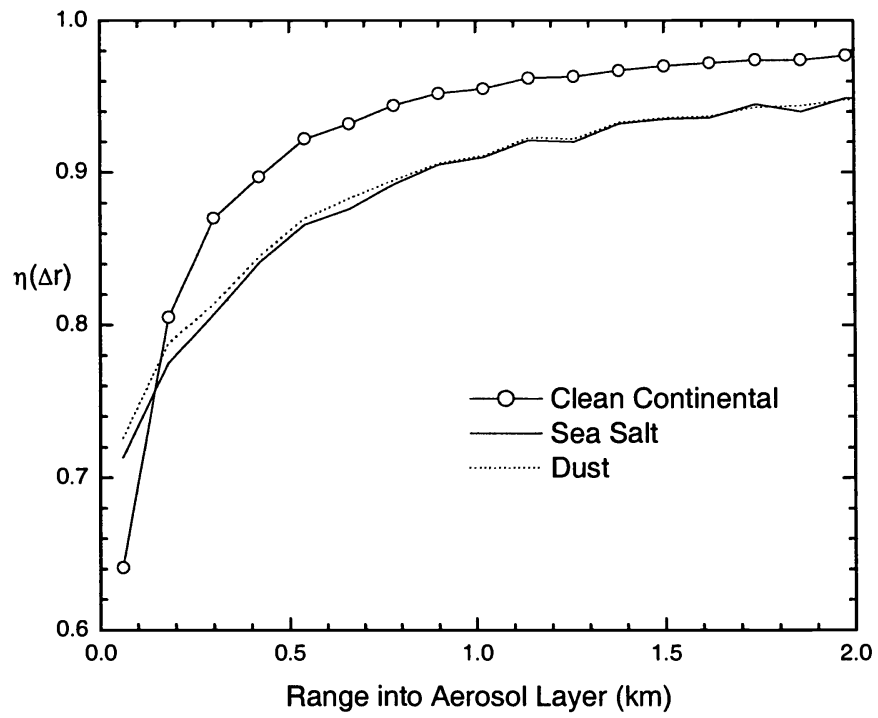

Figure 4. Range-dependent multiple scattering functions for three aerosol models, aerosol extinction $=0.2 / \mathrm{km}$.

\subsection{Ice clouds.}

In situ measurements show that cirrus clouds are composed of ice crystals having a wide variety of shapes and sizes. The shapes and sizes depend on the temperature, relative humidity, and dynamics of the layer. Additionally, ice crystal sizes and shapes change during the life-cycle processes of formation, sedimentation, and sublimation. To investigate the potential variability of $\eta(r)$ in ice clouds, two extreme cases are considered: tropical convective cirrus and polar cirrus. Phase functions derived from in situ measurements of vertical profiles of crystal size and shape within a tropical cirrus anvil are available from the CEPEX experiment. Phase functions were computed for several altitudes within the cloud using size-dependent combinations of irregular aggregates, bullet rosettes, hexagonal columns, and spheres ${ }^{14}$. The dependence of the phase functions on height within the cloud was found to be small. A phase function computed from measurements within the cloud is shown in Figure 5. Also shown in Figure 5 is a phase function representative of cold cirrus in the polar regions taken from Takano and Liou ${ }^{15}$, who based their calculation on regular hexagonal columns and a size distribution measured in cirrostratus. The crystal aspect ratio was varied with size according to observed behavior. It can be seen that the anvil cirrus, which is dominated by irregular particles, has a higher lidar ratio, weaker halo features, and significantly different side scattering compared to the regular hexagonal columns of the polar cirrus. 
When layer transmittance can be measured from the lidar return signal it is used as a constraint in performing the extinction retrievals. Otherwise, the CALIPSO Scene Classification Algorithm selects a cloud lidar ratio, $S_{c}$, to be used in the retrieval. For ice clouds, the value is selected using an empirically-derived relation between temperature and $S_{c}$. This approach requires that we have a predefined set of multiple scattering functions to correspond to each of the possible values of $S_{c}$.

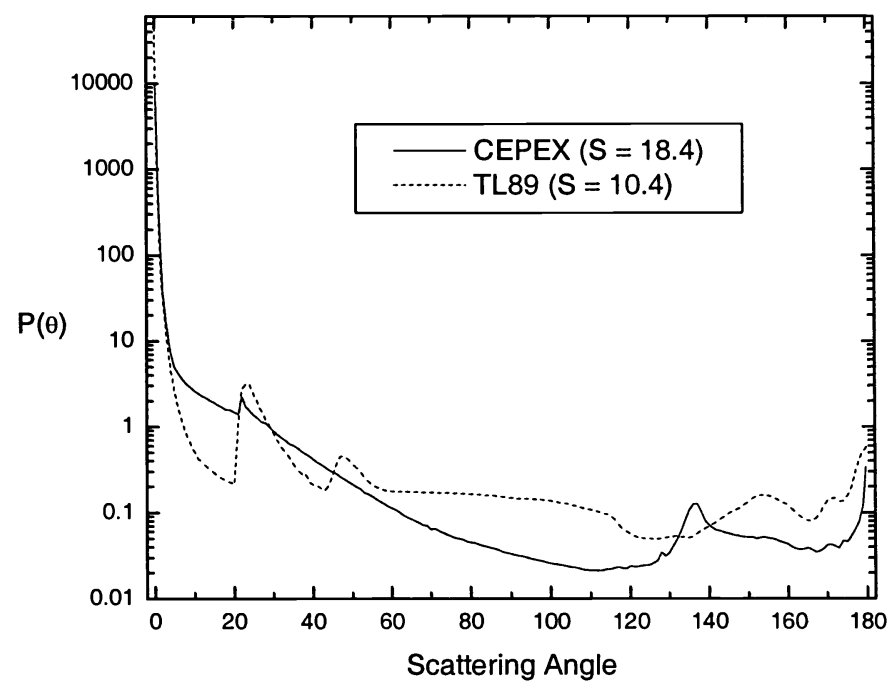

Figure 5. Cirrus phase functions for tropical convective $\operatorname{cirrus}^{14}$ (CEPEX) and polar cirrus ${ }^{15}$ (TL89).

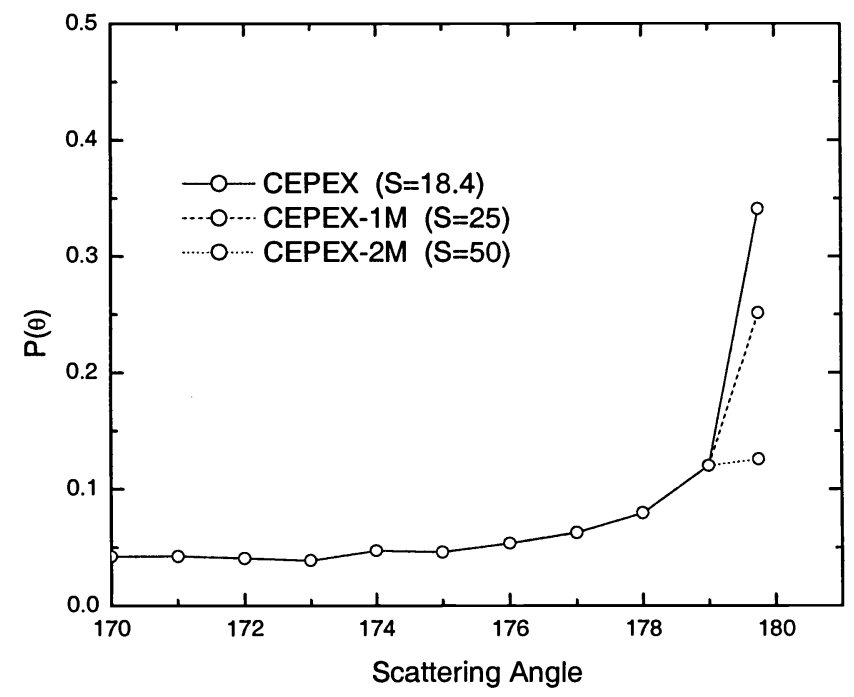

Figure 6. One CEPEX phase function modified to create several functions with different values of $S_{c}$.

Measurements of the cirrus lidar ratio ${ }^{16,17}$ show a range of values, with a mean value of about 25 sr and the majority of the values falling between $12 \mathrm{sr}$ and $50 \mathrm{sr}$. The lidar ratios of the phase functions shown in Figure 5 are low relative to these measured values. Ray tracing calculations show that $S_{c}$ increases significantly for hollow crystals ${ }^{18}$ and for 
irregular crystals ${ }^{19}$ relative to solid, regular crystals due to suppression of the backscatter. We can hypothesize that this is responsible for the higher values observed experimentally versus modeled values. Therefore, it is reasonable to create a family of phase functions which are consistent with a family of selected $S_{c}$ values by decreasing $\mathrm{P}(180)$ to give the desired values of $S_{c \text { c: }}$ Figure 6 shows two modifications of one of the CEPEX phase functions: CEPEX-1M with $S_{c}=25$, and CEPEX-2M with $S_{c}=50$.

The multiple scattering functions corresponding to the phase functions in Figures 5 and 6 are shown in Figure 7. In spite of the significant differences between the four phase functions, the $\eta(r)$ are seen to be quite similar and nearly independent of range. Thus it appears that, for cirrus, a single multiple scattering factor, $\eta_{c}$, can be used rather than a range-dependent function. The variation in $\eta(r)$ between different cirrus models is fairly small and suggests that errors in the retrieved extinction due to incorrect selection of the cirrus model will also be small.

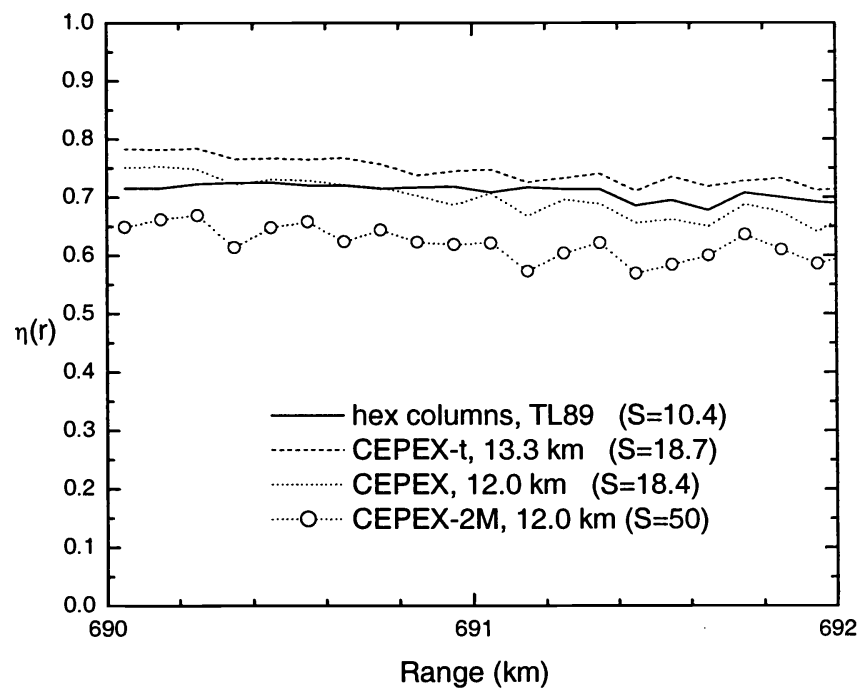

Figure 7. Range-dependent multiple scattering functions for cirrus.

The difference in the range-dependent behavior of $\eta(\mathrm{z})$ seen in Figure 4 and in Figure 7 is perhaps unexpected. Why is $\eta(\mathrm{z})$ nearly constant for cirrus, but increases with range into the layer for aerosols? Just beneath the top of the layer, a large range of scattering angles contribute to the double-scatter return signal. As the pulse penetrates into the layer, the portion of the double-scatter return signal due to large-angle scatters becomes smaller and smaller. For cirrus, most of the scatter is concentrated at small forward angles, and elimination of large-angle scatter has almost no effect on the return signal. The forward scattering peak for aerosols is much broader, so that elimination of large angle scattering does have an effect. As the pulse progresses into the layer, the exclusion of large-angle scattering significantly diminishes the relative magnitude of the multiple scattering and the magnitude of $\eta$ increases.

\section{APPLICATION}

Given the models for aerosol and cirrus multiple scattering presented above, the practical application of the extinction retrieval algorithm in specific situations is discussed.

\subsection{Optically thin clouds.}

When the two-way transmittance through the cloud can be measured accurately from the clear air return beneath the cloud layer, it can be used as a boundary condition for the extinction retrieval. This clear-air return is affected by 
multiple scattering within the cloud, however, and must be accounted for. The discussion in this section focuses on ice clouds. Although multiple scattering effects for optically thin water clouds are different, due to differences in particle size and shape, the same general considerations apply as those discussed here in the context of cirrus.

The primary multiple scattering effect in cirrus clouds is small-angle forward scattering due to the narrow diffraction patterns of ice crystals and the comparatively large receiver footprints of space lidars. This effect can greatly improve penetration in ice clouds but also means the forward scattering effects must be corrected to obtain measurements of cirrus transmittance and optical depth. Figure 8 shows $\eta(z)$ calculated using Equation (2) within and below a homogeneous cirrus layer with cloud top at a range of $695 \mathrm{~km}$ and cloud base at a range of $697 \mathrm{~km}$. Below cloud base, $\eta(\mathrm{z})$ is larger than within the cloud and nearly constant. This is because light scattered within the narrow forward diffraction peak of the ice crystals remains within the field of view of the lidar while large-angle scattering quickly

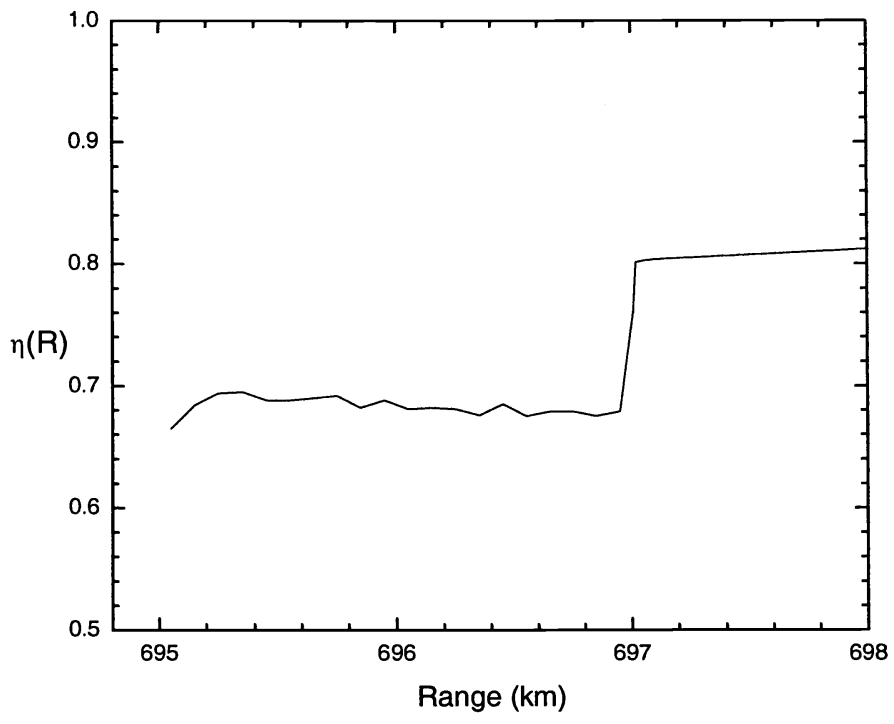

Figure 8. Multiple scattering function within and beneath a cirrus layer composed of regular hexagonal columns with cloud top at $695 \mathrm{~km}$ and base at $697 \mathrm{~km}$.

scatters out of the field of view. Because of this effect, the majority of the multiply-scattered signal below cloud base is from double scattering.

As described in Section 2, the algorithm for the retrieval of the cirrus backscatter requires a value of $S_{c} *$. When the 2way transmission through the cloud layer can be derived from the clear-air signal beneath the layer, it can be used together with the integrated attenuated backscatter to estimate $S_{c}{ }^{*}$. Given $S_{c}{ }^{*}$ we then retrieve $\beta(\mathrm{z})$. Finally, using a modeled estimate of $\eta_{c}$ to convert $S_{c} *$ to $S_{c}$, we obtain the extinction profile from: $\sigma(\mathrm{z})=S_{c} \beta(\mathrm{z})$.

$S_{c} *$ is estimated using an expression derived by Platt ${ }^{20}$ relating $S_{c} *$ to the layer transmittance, $\mathrm{T}^{2}=\exp \left(-2 \eta_{\mathrm{c}} \tau\right)$, and the layer-integrated backscatter, $\gamma^{\prime}$ :

$$
\mathrm{S}_{\mathrm{c}}^{*}=\left[1-\exp \left(-2 \eta_{\mathrm{c}} \tau\right)\right] / 2 \gamma^{\prime}
$$

However, Equation (3) is valid only within the cloud layer and the two-way transmission through the cloud that is actually measured from the clear-air return beneath the cloud is:

$$
\mathrm{T}^{, 2}=\exp \left(-2 \eta_{\mathrm{R}} \tau_{\mathrm{c}}\right),
$$


Here, $\eta_{c}$ is the value of the multiple scattering function within the cloud and $\eta_{R}$ is the value for clear air below the cloud. Platt ${ }^{20}$ assumed these were equal but this is not generally true, as seen above.

When $\eta_{\mathrm{c}} \neq \eta_{\mathrm{R}}$, the initial estimate of $S_{c}{ }^{*}$, denoted by $S_{c}{ }^{*}$, is:

$$
S_{c}{ }^{*}=\left[1-\exp \left(-2 \eta_{\mathrm{R}} \tau\right)\right] / 2 \gamma^{\prime}
$$

and the relative correction that must be applied is:

$$
\left(S_{c} *-S_{c}{ }^{*}\right) / S_{c} *=1-\left[1-\exp \left(-2 \eta_{\mathrm{R}} \tau\right)\right] /\left[1-\exp \left(-2 \eta_{\mathrm{c}} \tau\right)\right] .
$$

This correction factor is plotted in Figure 9 as a function of $\tau$. Values for $\eta_{R}$ and $\eta_{c}$ were estimated using multiple scattering calculations for cirrus and also for an elevated aerosol layer assumed to be mineral dust. The correction factors are seen to be relatively small and can be modeled with sufficient accuracy.

Given this estimate of $S_{c}{ }^{*}$, we can retrieve the attenuation-corrected backscatter profile, $\beta_{\mathrm{c}}(\mathrm{z})$, and finally the extinction profile:

$$
\sigma_{\mathrm{c}}(\mathrm{z})=S_{c} * \beta_{\mathrm{c}}(\mathrm{z}) / \eta_{\mathrm{c}}
$$

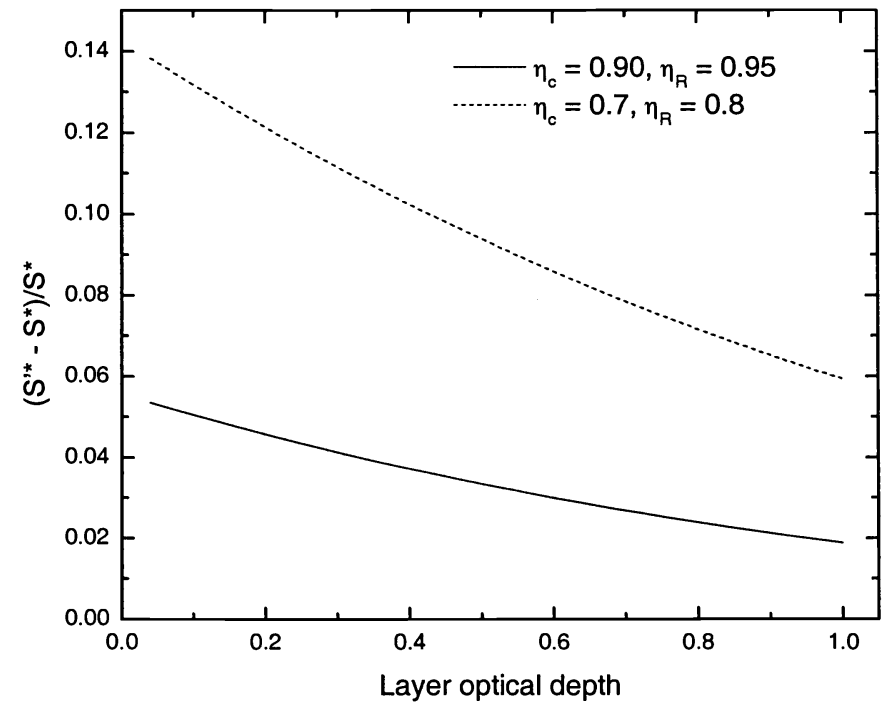

Figure 9. Relative correction to $\mathrm{S}^{\prime *}$ due to differences in the multiple scattering function within and below the layer: a) hexagonal columns (dashed line); b) mineral dust (solid line).

\subsection{Deep convective clouds}

Deep convective clouds often have tenuous tops so that the lidar signal penetrates a substantial distance, but does not penetrate to cloud base ${ }^{21}$. As above, we can use Equation (3) to obtain $S_{c}{ }^{*}$. Because the transmission term is zero in this case, Equation (3) reduces simply to: 


$$
\mathrm{S}_{\mathrm{c}}^{*}=1 / 2 \gamma^{\prime}
$$

CEPEX results show little variation in $S_{c}$ or $\eta_{\mathrm{c}}$ with height within the cloud ${ }^{14}$, so $S_{c}{ }^{*}$ can be assumed to be constant with range. Once $S_{c}{ }^{*}$ has been derived, the attenuation-corrected backscatter profile, $\beta_{\mathrm{c}}(\mathrm{z})$ can be retrieved. Finally, $\eta_{\mathrm{c}}$ is needed to convert $\sigma_{\mathrm{c}}(\mathrm{z})$ to $\beta_{\mathrm{c}}(\mathrm{z})$. As shown in Figure $7, \eta_{\mathrm{c}}$ shows little variation in tropical convective cirrus and the extinction profile can be retrieved as for thin cirrus:

$$
\sigma_{\mathrm{c}}(\mathrm{z})=S_{c} * \beta_{\mathrm{c}}(\mathrm{z}) / \eta_{\mathrm{c}}
$$

\subsection{Very tenuous clouds.}

Clouds which have very low optical depths will have a two-way transmittance too close to unity to measure accurately. For these clouds, we must use modeled values for both $S_{c}$ and $\eta_{c}(\mathrm{r})$, since we have no transmittance information. The multiple scattering factor and $S_{c}$ must be estimated in a consistent manner, however. Since the optical depths of these clouds is quite low ( $\tau$ on the order of 0.2 or less), large relative errors in optical depth are still small in an absolute sense.

\subsection{Aerosols}

Aerosols are usually more tenuous than clouds, with extinction coefficient typically less than $1 / \mathrm{km}$ except near strong sources. Because aerosol layers usually extend to the surface, we rarely have the layer transmittance to use as a constraint and so, in terms of a retrieval, must treat them similar to the very tenuous clouds discussed above.

The same extinction retrieval algorithm is used for aerosol as for clouds. So once again a value of $\mathrm{S}^{*}$ is needed to retrieve the aerosol backscatter profile. For aerosol layers, the CALIPSO Scene Classifier Algorithm determines the most likely aerosol type, prior to the extinction retrieval, based on lidar observables and other information. Once an aerosol model is specified, the values of $S_{a}$ and $\eta(z)$ defined by that model are used to construct

$$
\mathrm{S}^{*}(\mathrm{z})=\eta(\mathrm{z}) \mathrm{S}_{\mathrm{a}} \text {. }
$$

$S_{a}$ is assumed by the algorithm to be constant within the layer. The attenuation-corrected aerosol backscatter profile can then be retrieved and finally the aerosol extinction profile, $\sigma_{\mathrm{a}}(\mathrm{z})$, is determined from $\sigma_{\mathrm{a}}(\mathrm{z})=S_{a} \beta(\mathrm{z})$.

\section{SUMMARY}

A straightforward method of accounting for multiple scattering effects in the retrieval of extinction profiles from satellite lidar has been presented. The most difficult aspect is the development of appropriate multiple scattering functions, based on realistic aerosol and cloud models, required by the retrieval algorithm. Recent field campaigns have provided new measurements of aerosol and cloud properties allowing improved estimates of the multiple scattering function. Validation of the estimated multiple scattering functions using coincident correlative measurements acquired during the on-orbit phase of the CALIPSO mission will be critical, however.

\section{ACKNOWLEDGEMENTS}

I would like to thank Greg McFarquhar and Irina Sokolik for providing some of the phase functions used in this study.

\section{REFERENCES}

1. D. Gambling and K. Bartusek, "Lidar observations of tropospheric aerosols," Atmos. Env. 6, pp. 181-190, 1972. 
2. F. G. Fernald, B. M. Herman, and J. A. Reagan, "Determination of aerosol height distributions by lidar," J. Appl. Meteor. 11, pp. 482-499, 1972.

3. J. D. Klett, "Stable Analytical Inversion Solution for Processing Lidar Returns," Appl. Opt. 20, p. 211, 1981.

4. D. M. Winker, R. H. Couch, and M. P. McCormick, 'An overview of LITE: NASA's Lidar In-space Technology Experiment," Proc. IEEE 84, pp. 164-180, 1996.

5. D. M. Winker, "Simulation and modeling of multiple scattering effects observed in LITE data." in Advances in Atmospheric Remote Sensing with Lidar, A. Ansmann, R. Neuber, P. Rairoux, and U. Wandinger, eds., pp. 185-188, (Springer Verlag, Berlin), 1997.

6. D. M. Winker, J. Pelon, and M. P. McCormick, "The CALIPSO mission: Spaceborne lidar for observation of aerosols and clouds," Proc. SPIE vol. 4893, in press, 2002.

7. D. M. Winker and L. R. Poole, "Monte-Carlo calculations of cloud returns for ground-based and space-based lidars," Phys Rev. B 60, pp. 341-344, 1995.

8. Y.-X. Hu, D. M. Winker, P. Yang, B. Baum, L. R. Poole, and L. Vann, "Identification of cloud phase from PICASSO-CENA lidar depolarization: a multiple scattering sensitivity study," J. Quant. Spec. Rad. Trans. 70, pp. 569-579, 2001.

9. S. A. Young, M. A. Vaughan, and D. M. Winker, "Adaptive methods for retrieving extinction profiles from space applied to CALIPSO lidar data," in Lidar Remote Sensing in Atmospheric and Earth Sciences: Proceedings of the $21^{\text {st }}$ ILRC, L. Bissonnette, G. Roy, and G. Vallee, eds., pp. 743-746, (Defence R\&D Canada - Valcartier, Quebec, Canada), 2002.

10. C. M. R. Platt, "Remote sounding of high cirrus clouds. III: Monte Carlo calculations of multiple-scattered lidar returns," J. Atmos. Sci. 38, 156-167, 1981.

11. W. Carnuth and R. Reiter, "Cloud extinction profile measurements by lidar using Klett's inversion method," Appl. Opt. 25, pp. 2899-2907, 1986.

12. S. J. Masonis, T. L. Anderson, and D. S. Covert, "A study of the extinction to backscatter ratio and its relation to other aerosol optical properties during the Shoreline Environment Aerosol Study, with a comparison to a polluted site and to Mie theory," submitted to J. Atmos. Ocean. Tech., 2002.

13. O. V. Kalashnikova and I. N. Sokolik, "Importance of shapes and compositions of wind-blown dust particles for remote sensing at solar wavelengths," Geophys. Res. Lett. in press, 2002.

14. G. M. McFarquhar, A. J. Heymsfield, A. Macke, J. Iaquinta, S. M. Aulenbach, "Use of observed ice crystal sizes and shapes to calculate mean-scattering properties and multispectral radiances: CEPEX April 4, 1993, case study," J. Geophys. Res. 104, pp. 31 763-31 779, 1999.

15. Y. Takano and K. N. Liou, "Solar radiative transfer in cirrus clouds. Part I: Single-scattering and optical properties of hexagonal ice crystals," J. Atmos. Sci. 46, pp. 3-18, 1989.

16. K. Sassen and J. M. Comstock, "A midlatitude cirrus cloud climatology from the facility for atmospheric remote sensing. Part III: Radiative properties," J. Atmos. Sci. 58, pp. 2113-2127, 2001.

17. E. W. Eloranta, R. E. Kuehn, and R. E. Holz, "Measurements of Backscatter Phase Function and Depolarization in Cirrus Clouds made with the University of Wisconsin High Spectral Resolution Lidar" in Advances in Laser Remote 
Sensing: Selected Papers Presented at the $20^{\text {th }}$ International Laser Radar Conference, Vichy, France, July 2000, A. Dabas, C. Loth, J. Pelon, eds., pp. 255-258 (Editions de l'Ecole Polytechnique, Palaiseau, France), 2001.

18. Y. Takano and K. N. Liou, "Solar Radiative Transfer in Cirrus Clouds. Part I: Single-scattering and Optical Properties of Hexagonal Ice Crystals," J. Atmos. Sci. 46, pp. 3-19, 1989.

19. A. Macke, J. Mueller, and E. Raschke, "Single scattering properties of atmospheric ice crystals," J. Atmos. Sci. 53, pp. 2813-2825, 1996.

20. C. M. R. Platt, "Lidar and radiometric observations of cirrus clouds," J. Atmos. Sci. 30, pp. 1191-1204, 1973.

21. C. M. R. Platt, D. M. Winker, M. A. Vaughan, and S. D. Miller, "Backscatter-to-extinction ratios in the top layers of tropical mesoscale convective systems and in isolated cirrus from LITE observations," J. Appl. Meteor. 38, pp. 1330-1345, 1999. 